\title{
Effects of Training Attendance on Muscle Strength of Young Men after
}

\author{
11 Weeks of Resistance Training
}

Paulo Gentil, PhD*ABCDEG ; Martim Bottaro, PhD ${ }^{\text {ABDEFG }}$

\author{
Authors' Affiliation: \\ College of Physical Education, University \\ of Brasilia, Brasilia, Brazil College of \\ Health Science, University of Brasilia \\ Brazil
}

\begin{abstract}
Purpose: Training attendance is an important variable for attaining optimal results after a resistance training (RT) program, however, the association of attendance with the gains of muscle strength is not well defined. Therefore, the purpose of the present study is to verify if attendance would affect muscle strength gains in healthy young males.
\end{abstract}

Methods: Ninety two young males with no previous RT experience volunteered to participate in the study. RT was performed 2 days a week for 11 weeks. One repetition maximum (1RM) in the bench press and knee extensors peak torque (PT) were measured before and after the training period. After the training period, a two step cluster analysis was used to classify the participants in accordance to training attendance, resulting in three groups, defined as high (92 to $100 \%$ ), intermediate ( 80 to $91 \%$ ) and low $(60$ to $79 \%$ ) training attendance.

Results: According to the results, there were no significant correlations between strength gains and training attendance, however, when attendance groups were compared, the low training attendance group showed lower increases in 1RM bench press $(8.8 \%)$ than the other two groups $(17.6 \%$ and $18.0 \%$ for high and intermediate attendance, respectively).

Conclusions: Although there is not a direct correlation between training attendance and muscle strength gains, it is suggested that a minimum attendance of $80 \%$ is necessary to ensure optimal gains in upper body strength.

Key Words: Compliance; Strength Training; Resistance Exercise; Bench Press; Knee Extensors; Peak Torque

\section{INTRODUCTION}

$\mathrm{I}^{\mathrm{n}}$ $\mathrm{n}$ order to attain optimal results in any physical activity program, it is important to alternate training sessions with adequate periods of rest. This interaction between load and recovery is the basis of the supercompensation phenomenon ${ }^{[1]}$ that also applies to resistance training (RT). During an RT session the load induces alterations in the neuromuscular system, with the occurrence of muscle damage and fatigue ${ }^{[2]}$. It is expected that during the following days, the system recovers its capacity and a small positive effect is elicited. In long term, the sum of these small positive effects would lead to strength gains as a result of continuous RT ${ }^{[3]}$. However it is important to note that protein synthesis ${ }^{[4-9]}$ and glucose absorption ${ }^{[10-12]}$ may return to basal levels after few days or even hours after the training session. Therefore, the RT stimulus must be repeated in an adequate time, in order to attain chronic improvements in muscle function.

Due to the transitory characteristics of the morphofunctional alterations induced by a training session, a low training attendance may alter the balance between training and recovery, hindering long-term results. It is common among studies using RT to establish a minimum training attendance that is usually defined between 80 to $90 \%$, because it is believed that this is the minimum attendance necessary to attain the 
expected results ${ }^{[13-15]}$.

In fact, previous studies have proven associations between RT attendance and body composition changes in diabetics ${ }^{[16]}$ and post menopausal women ${ }^{[17]}$, reduction in depressive symptoms ${ }^{[18]}$, and improvements on quality of life scores in older people ${ }^{[19]}$. However, we are unaware of any published studies analyzing the effect of attendance on muscle strength in young people. Furthermore, given the high popularity of RT and the increasing number of researches using this mode of exercise, it would be important to clarify the effects of training attendance in order to allow an optimal RT program design. Therefore, the purpose of the present study is to verify if increasing attendance would increase muscle strength gains in health young males performing RT. Our hypothesis is that attendance would be related to strength gains in response to RT.

\section{METHODS AND SUBJECTS}

\section{Subjects:}

Ninety two college aged men volunteered to participate in the study $(21.7 \pm 2.7$ years; $175.4 \pm 6.7 \mathrm{~cm} ; 74.1 \pm$ $15.0 \mathrm{~kg}$ ). Volunteers were selected at random from respondents to fliers distributed over the university campus, and by word-of-mouth. The criteria for entering the study included being at least 18 years of age, having no previous RT experience and being free of clinical problems that could be aggravated by the study procedures. Evaluation of food intake was made by a 3 day recordatory inquiry. The volunteers were oriented to not change their nutritional habits during the study period, and if any relevant change was detected (i.e. becoming a vegetarian, restricting calories, taking nutritional supplements or ergogenic aids, etc.) the participants' data were excluded from the analysis.

All participants were notified of the research procedures, requirements, benefits and risks before providing written informed consent. The Institutional Research Ethics Committee granted approval for the study.

\section{One repetition maximum (1RM) test:}

In the week before the experiment and 5-7 days after the last training session, the load for 1RM was determined for each subject in the bench press exercise using the protocol suggested by Kraemer and Fry ${ }^{[20]}$. The initial tests were repeated in all subjects and data were analyzed by Pearson product moment correlations to estimate day-to-day $1 \mathrm{RM}$ reliability $(\mathrm{r}=0.93)$. Also, no significant differences were found between day-today 1RM loads.

\section{Isokinetic concentric peak torque (PT):}

Knee extensor isokinetic concentric PT was measured on a Biodex System 3 Isokinetic Dynamometer (Biodex Medical, Inc., Shirley, NY). Calibration of the dynamometer was performed according to the manufacturer's specifications before every testing session. The subjects sat upright with the axis of rotation of the dynamometer arm oriented with the lateral femoral condyle of the right knee. Belts were used to secure the thigh, pelvis, and trunk to the dynamometer chair to prevent additional body movement. The chair and dynamometer settings were recorded to ensure the same positioning for all tests. Gravity correction was obtained by measuring the torque exerted on the dynamometer resistance adapter with the knee in a relaxed near full extension. The tests comprised two sets of five repetitions at $60 \%$ s. Subjects were instructed to fully extend and flex the knee and to work maximally during each set. Verbal encouragement was given throughout the test session. After each set, subjects were required to take $60 \mathrm{~s}$ of rest before the onset of the next set. The knee strap was released during each rest period to ensure unrestricted blood flow to the lower limb. The procedures were administered to all subjects by the same investigator. Knee extensor PT baseline test and retest ICC and standard error of the mean (SEM) were 0.98 and $2.3 \%$ respectively.

\section{Resistance training protocol:}

Resistance training sessions consisted of five exercises including leg press, knee flexion, bench press, pull down and sit ups. Subjects performed two sets of 8-12 repetitions, with 90-120 second intervals between sets and exercises, respectively. All sets were performed 
until concentric failure. Resistance was increased by the exercise technician when a subject completed 12 repetitions for at least one of the two sets while maintaining proper form. All participants followed the same resistance training program for 11 weeks under supervision. Training sessions were closely supervised by experienced trainers, because previous research has demonstrated greater gains in supervised vs. unsupervised training ${ }^{[21]}$.

Training was conducted two days a week, with a minimum of 48 hours between sessions. Each subject was instructed to record training logs for each workout day. All training $\operatorname{logs}$ for the 11 -week study were completed and verified by a researcher/supervisor following each exercise session.

The volunteers were randomly divided into 4 groups of no more than 25 individuals. Training attendance was only defined after final tests, and the attendance groups were defined using cluster analysis.

\section{Statistical procedures:}

Normal distribution of the data was confirmed using the Kolmogorov-Smirnov tests. Data are presented as mean \pm standard deviation. Attendance was defined as the number of sessions attended divided by the number offered, multiplied by $100 \%$. 1RM load for bench press and knee extensor PT are shown in scatter plots (figures 1 and 2, respectively), with the attendance values in the $\mathrm{X}$ axis. The Pearson linear correlation was calculated to analyze the association between strength gains and training attendance.
The two-step cluster analysis was used to create homogenous groups according to training attendance. Cluster analysis was used to minimize within-group and maximizing between-group variations. Although the use of fixed values is common, it may create heterogeneous groups, with relatively large withingroup variations.

After group definition, paired $\mathrm{t}$ tests were used to calculate within group differences between pre and post tests. Analysis of covariance (ANCOVA) was used to compare post training 1RM bench press and knee extensor PT among attendance groups, using baseline values as covariates. The Bonferroni procedure was used for post hoc analysis. The probability level of statistical significance was set at $\mathrm{P}<$ 0.05 in all comparisons. Data were analyzed using the SPSS 16.0 (SPSS Inc., Chicago, IL, USA) statistical software package.

\section{RESULTS}

Alterations in 1RM bench press and knee extensor PT in relation to training attendance are illustrated in figures 1 and 2, respectively. The results of Pearson correlation did not show significant correlation $(P>$ $0.05)$ between attendance and 1RM changes $(\mathrm{r}=-0,2)$ and PT gains $(\mathrm{r}=0.2)$.

Cluster analysis lead to the formation of three

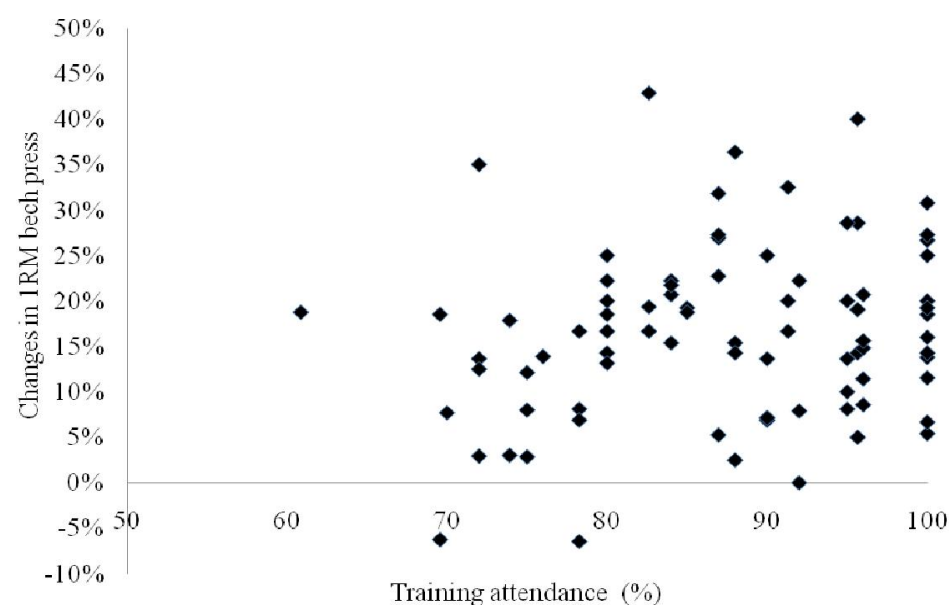

Fig. 1: changes in 1RM bench press in relation to training attendance 


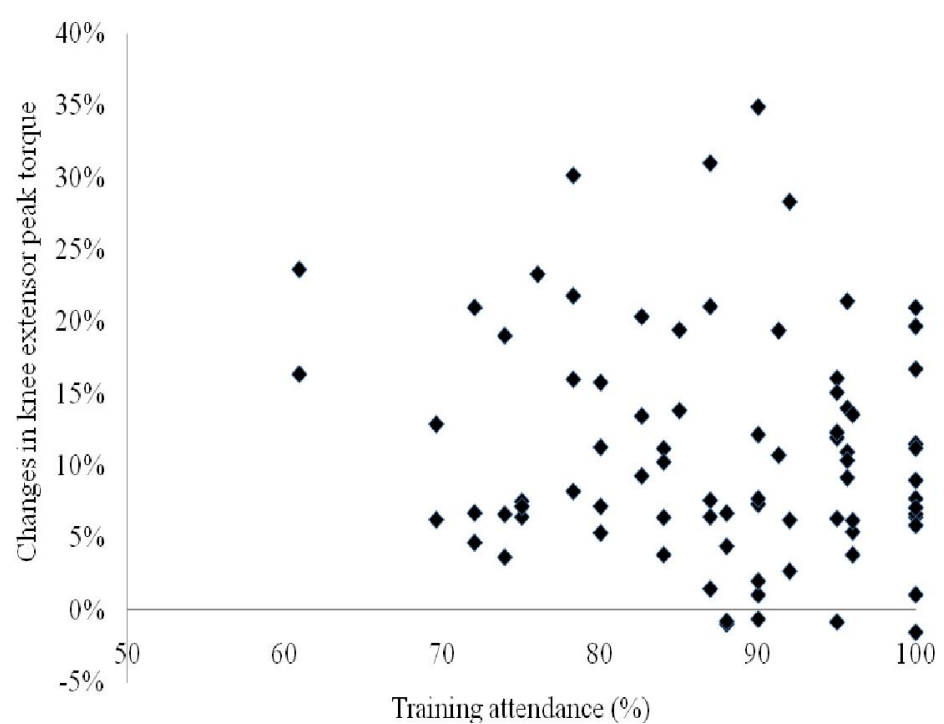

Fig. 2: changes in knee extensor peak torque in relation to training attendance

groups in accordance with attendance levels: high attendance (92 to $100 \%$ ), intermediate attendance ( 80 to $91 \%$ ) and low attendance (60 to $79 \%$ ). Subject's characteristics, according to each group, are reported in table 1.

Within groups analysis revealed that all training attendance groups showed significant increases in knee extensor PT and 1RM bench press $(P>0.05)$. ANCOVA showed between groups differences for post training 1RM bench press, indicating that the low attendance group had lower values than the other two groups $(P<0.05)$. There were no differences for the knee extensors PT $(P>0.05)$.

\section{DISCUSSION}

Contrary to our hypothesis, the results of the present study showed no correlation between training attendance and strength gains after RT in young men, therefore, it is not possible to suggest that, in the initial training phase, a higher attendance would lead to better results. However, there seems to exist a cutting point, from where the lack of compliance starts to hinder upper body strength gains. In the present study, the group of subjects with less than $80 \%$ of training attendance showed less gains in 1RM bench press than the other groups. Interestingly, there were no

Table 1: Characteristics of the subjects according to the attendance groups

\begin{tabular}{|c|c|c|c|}
\hline Parameter & $\begin{array}{l}\text { High attendance } \\
\qquad(\mathbf{N}=\mathbf{3 5})\end{array}$ & $\begin{array}{l}\text { Intermediate attendance } \\
\qquad(\mathbf{N}=\mathbf{3 6})\end{array}$ & $\begin{array}{l}\text { Low attendance } \\
\qquad(\mathrm{N}=\mathbf{2 1})\end{array}$ \\
\hline Attendance (\%) & $96.9(2.8)$ & $85.7(3.9)$ & $72.9(4.9)$ \\
\hline Age (years) & $21.3(2.6)$ & $21.39(2.2)$ & $23.2(3.5)$ \\
\hline Height (cm) & $175.7(6.9)$ & $175.18(6.5)$ & $175.4(6.8)$ \\
\hline Weight (kg) & $71.3(13.6)$ & $73.6(16.9)$ & $71.1(14.2)$ \\
\hline \multicolumn{4}{|l|}{ Knee extensors peak torque } \\
\hline Pre (N.m) & $224.6(38.8)$ & $215.6(36.4)$ & $204.4(35.0)$ \\
\hline Post (N.m) & $249.4(38.2)^{\neq}$ & $237.2(35.1)^{\neq}$ & $229.4(36.6)^{\neq}$ \\
\hline Delta $(\%)$ & 11.1 & 10.0 & 12.2 \\
\hline \multicolumn{4}{|l|}{ 1RM bench press } \\
\hline $\begin{array}{r}\text { Pre (kg) } \\
\text { Post (kg) } \\
\text { Delta (\%) }\end{array}$ & $\begin{array}{c}57.3(11.7) \\
67.4(10.97)^{f} \\
17.6^{*}\end{array}$ & $\begin{array}{c}55.3(12.7) \\
65.2(13.7)^{\neq} \\
\quad 18.0 *\end{array}$ & $\begin{array}{c}61.6(10.1) \\
67(10.9)^{\neq} \\
8.8\end{array}$ \\
\hline
\end{tabular}

1RM: one repetition maximum, $* P<0.05$ vs. low attendance group; $\neq P<0.05$ vs. Pre 
differences between the attendance groups for gains in lower body strength.

According to the results of the nutritional analysis the participants in the present study reached the protein ingestion recommended by most authors ${ }^{[22-24]}$ and no significant changes in the nutritional habits were observed. Therefore, nutritional habits probably did not influence the results.

RT induces acute alterations, like muscle damage and fatigue, that lead to decreases in muscle strength and the initiation of inflammatory processes ${ }^{[2,25]}$. After the training session and in the presence of adequate nutrition and recovery, an anabolic phase starts, with the recovery of muscle capability and the occurrence of a small positive effect ${ }^{[3,26]}$. The sum of these small positive effects will lead, in long term, to an increase in muscle size and strength. Therefore, training repetition is supposed to be important for long term results, and a low training attendance may impair the training effects. However, although the importance of training attendance is well accepted, there is no definition of the values necessary to get optimal results during the earlier stages of RT.

A 14 week study indicated that an attendance higher than $75 \%$ is important to reduce waist circumference in diabetics ${ }^{[16]}$. In older women it has been shown that, in a six month period, training attendance affects the results of a quality of life questionnaire ${ }^{[19]}$. With regard to body composition, Bea et al. ${ }^{[17]}$ studied post menopausal women over 6 months of training and verified that fat free mass increased only in the volunteers with higher training attendance ( $>67 \%$ ).

With regard to strength gains, the study of Chin et al ${ }^{[13]}$ did not find any difference for upper of lower body strength between the control group and the group that did RT for 6 months. The authors attributed the lack of results to the low training attendance that averaged $76 \%$. Although our study had a shorter duration, we found significant gains in strength in all groups. These increases even in the low attendance group seen in the present study may be due to the characteristics of the subjects, since the study of Chin et al. was done on frail elderlies and the present study was performed on healthy young men. However, it is interesting to note the relation of the attendance values that compromise the strength gains; in the present study, results were comprised for attendance lower than $80 \%$ and the attendance was $75 \%$ in Chien's study.

\section{CONCLUSION}

Based on the results of the present study, we suggest that, during exercise prescription for young men, it is recommended to establish a minimum of $80 \%$ of training attendance to get optimal upper body strength gains. This information may be important for coaches and athletes during the design and execution of an RT program and for researches in the design of research protocols.

One limitation of the present study was that there were no individuals with very low training attendance $(<60 \%)$, which limited a broader analysis. One suggestion for future researches is to study the effects of training attendance on other muscle adaptations, such as muscle hypertrophy.

\section{ACKNOWLEDGMENT}

The permission of this study was obtained from the University of Brasilia Institutional Review Board.

Conflict of interests: None

\section{REFERENCES}

[1] Issurin VB. New horizons for the methodology and physiology of training periodization. Sports Med 2010;40:189-206.

[2] Flores DF, Gentil P, Brown LE, et al. Dissociated time course of recovery between genders after resistance exercise. J Strength Cond Res 2011;25:3039-44. 
[3] Yarasheski KE. Exercise, aging, and muscle protein metabolism. J Gerontol A Biol Sci Med Sci 2003;58:M918-22.

[4] Chesley A, MacDougall JD, Tarnopolsky MA, et al. Changes in human muscle protein synthesis after resistance exercise. $J$ Appl Physiol 1992;73:1383-8.

[5] MacDougall JD, Gibala MJ, Tarnopolsky MA, et al. The time course for elevated muscle protein synthesis following heavy resistance exercise. Can J Appl Physiol 1995;20:480-6.

[6] Tipton KD, Ferrando AA, Phillips SM, et al. Postexercise net protein synthesis in human muscle from orally administered amino acids. Am J Physiol Endocrinol Metab 1999;276:E628-E34.

[7] Phillips SM, Tipton KD, Aarsland A, et al. Mixed muscle protein synthesis and breakdown after resistance exercise in humans. Am J Physiol 1997;273:E99-107.

[8] Burd NA, Groen BB, Beelen M, et al. The reliability of using the single-biopsy approach to assess basal muscle protein synthesis rates in vivo in humans. Metabolism 2012;61:931-6.

[9] Burd NA, Andrews RJ, West DW, et al. Muscle time under tension during resistance exercise stimulates differential muscle protein sub-fractional synthetic responses in men. $J$ Physiol 2012;590:351-62.

[10] Koopman R, Manders RJF, Zorenc AHG, et al. A single session of resistance exercise enhances insulin sensitivity for at least $24 \mathrm{~h}$ in healthy men. Eur J Appl Physiol 2005;94:180-87.

[11] Biolo G, Williams BD, Fleming RY, et al. Insulin action on muscle protein kinetics and amino acid transport during recovery after resistance exercise. Diabetes 1999;48:949-57.

[12] Hansen E, Landstad BJ, Gundersen KT, et al. Insulin sensitivity after maximal and endurance resistance training. J Strength Cond Res 2012;26:327-34.

[13] Chin APMJ, van Poppel MN, Twisk JW, et al. Once a week not enough, twice a week not feasible? A randomised controlled exercise trial in long-term care facilities [ISRCTN87177281]. Patient Educ Couns 2006;63:205-14.

[14] Gentil P, Pereira RW, Leite TK, et al. ACTN3 R577X polymorphism and neuromuscular response to resistance training. J Sports Sci Med 2011;10:393-99.

[15] Kadoglou NP, Fotiadis G, Athanasiadou Z, et al. The effects of resistance training on ApoB/ApoA-I ratio, Lp(a) and inflammatory markers in patients with type 2 diabetes. Endocrine. 2012;42:561-9.

[16] Sukala WR, Page R, Rowlands DS, et al. South Pacific Islanders resist type 2 diabetes:comparison of aerobic and resistance training. Eur J Appl Physiol 2012;112:317-25.

[17] Bea JW, Cussler EC, Going SB, et al. Resistance training predicts 6-yr body composition change in postmenopausal women. Med Sci Sports Exerc 2010;42:1286-95.

[18] Baker MK, Kennedy DJ, Bohle PL, et al. Efficacy and feasibility of a novel tri-modal robust exercise prescription in a retirement community:a randomized, controlled trial. J Am Geriatr Soc 2007;55:1-10.

[19] Liu-Ambrose TY, Khan KM, Eng JJ, et al. Both resistance and agility training reduce back pain and improve health-related quality of life in older women with low bone mass. Osteoporos Int 2005;16:1321-9.

[20] Kraemer WJ, Fry AC. Strength testing:development and evaluation of methodology. In:Maud P, Foster C (eds). Physiological Assessment of Human Fitness. Champaingn, IL:Human Kinetics. 1995; Pp:115-38.

[21] Gentil P, Bottaro M. Influence of supervision ratio on muscle adaptations to resistance training in nontrained subjects. J Strength Cond Res 2010;24:639-43.

[22] Lemon PW, Tarnopolsky MA, MacDougall JD, et al. Protein requirements and muscle mass/strength changes during intensive training in novice bodybuilders. J Appl Physiol 1992;73:767-75.

[23] Iglay HB, Thyfault JP, Apolzan JW, et al. Resistance training and dietary protein:effects on glucose tolerance and contents of skeletal muscle insulin signaling proteins in older persons. Am J Clin Nutr 2007;85:1005-13.

[24] Dohm GL. Protein nutrition for the athlete. Clin Sports Med 1984;3:595-604.

[25] Hawke TJ, Garry DJ. Myogenic satellite cells:physiology to molecular biology. J Appl Physiol 2001;91:534-51.

[26] Folland JP, Williams AG. The adaptations to strength training :morphological and neurological contributions to increased strength. Sports Med 2007;37:145-68. 